\title{
Semilinear Cauchy Problems with Almost Sectorial Operators
}

by

\author{
Tomasz DLOTKO
}

Presented by Jerzy ZABCZYK

Dedicated to the memory of Professor Andrzej Lasota

Summary. Existence of a mild solution to a semilinear Cauchy problem with an almost sectorial operator is studied. Under additional regularity assumptions on the nonlinearity and initial data we also prove the existence of a classical solution to this problem. An example of a parabolic problem in Hölder spaces illustrates the abstract result.

1. Introduction. The aim of this paper is to study existence and regularity of solutions to the general semilinear Cauchy problem

$$
u_{t}+A u=f(t, u), \quad u(0)=u_{0},
$$

in a Banach space $X$, where $A: D(A) \subset X \rightarrow X$ generates an integrated semigroup of a special kind. More precisely, we will assume $A$ to be a closed linear operator whose spectrum $\sigma(A)$ is contained in the sector $S_{\omega}=$ $\{z \in \mathbb{C} \backslash\{0\}:|\arg z| \leq \omega\} \cup\{0\}$ for some $0<\omega<\pi / 2$ and whose resolvent $\varrho(A)$ satisfies: for every $\omega<\eta<\pi$ there is a constant $C_{\eta}>0$ such that

$$
\left\|(\lambda-A)^{-1}\right\| \leq C_{\eta}|\lambda|^{\gamma} \quad \text { for all } \lambda \in \mathbb{C} \backslash S_{\eta} \text { and some } \gamma \in(-1,0) .
$$

Such operators will be called almost sectorial. It turns out that this definition implies that $0 \in \varrho(A)$ (see [P-S, Remark 2.2]).

The notion of "integrated semigroup" was introduced in 1983 by W. Arendt [A1] and studied in a number of later references (e.g. [A2], [K-H], $[\mathrm{N}-\mathrm{S}],[\mathrm{P}],[\mathrm{P}-\mathrm{S}],[\mathrm{T}])$. This theory is an extension of the classical theory of

2000 Mathematics Subject Classification: 35A07, 35K90, 37L05.

Key words and phrases: Cauchy problem, integrated semigroup, almost sectorial operator, mild solution, classical solution. 
$C^{0}$-semigroups. Properties of integrated semigroups are different from those of $C^{0}$-semigroups (a list of such properties can be found in $[\mathrm{T}]$ ).

Below we will formulate certain existence theorems for the semilinear problem (1.1) involving an almost sectorial operator. Such problems generate semigroups with properties intermediate between those of $C^{0}$ and integrated semigroups. In the main body of the paper, under appropriate assumptions on the nonlinear term $f$, we will show the existence of a local mild solution to (1.1). This solution will have additional smoothness, in particular will be a classical solution, for regular initial data $u_{0}$.

Results of that kind were already reported in $[\mathrm{P}],[\mathrm{P}-\mathrm{S}]$ and $[\mathrm{A}-\mathrm{C}-\mathrm{L}]$. In the interesting paper [P-S] the McIntosh functional calculus for almost sectorial operators was built, and local and global solvability of the nonhomogeneous linear Cauchy problem with an almost sectorial operator was studied. An extension of the existence theory to semilinear equations was reported later in $[\mathrm{P}]$. Our assumptions and results overlap with those of $[\mathrm{P}]$. However, our definition of the mild solution (following [A-C-L]) is different; in Theorem 2 we study additionally existence of classical solutions; we also discuss in detail an example of a semilinear parabolic Cauchy problem in Hölder spaces, which was our main motivation for writing this paper (see $[\mathrm{FR}]$ for classical results concerning this example).

2. Linear semigroup. Let $(X,\|\cdot\|)$ be a complex Banach space. It was shown in $[\mathrm{P}-\mathrm{S}]$ that even without assuming the domain $D(A)$ to be dense in $X$, the part of $-A$ in the Banach space $(\overline{D(A)},\|\cdot\|)$ is the complete generator of an analytic semigroup of growth order $\gamma+1,\{T(t): t \in \mathbb{C} \backslash\{0\}$, $|\arg t|<\pi / 2-\omega\}$, which is given by

$$
T(t)=\frac{1}{2 \pi i} \int_{\Gamma_{\theta}} e^{-t z}(z-A)^{-1} d z,
$$

where $\Gamma_{\theta}=\left\{r e^{-i \theta}: r>0\right\} \cup\left\{r e^{i \theta}: r>0\right\}$ with $\omega<\theta<\eta<\pi$ is oriented counter-clockwise. The $T(t)$ defined by (2.1) have the semigroup property $T(t) T(s)=T(t+s), t, s>0$, which is verified in the usual way by calculating contour integrals and using the resolvent identity. It also follows from (1.2) that

$$
\|T(t)\| \leq M t^{-(\gamma+1)} \quad \text { for all } t>0,
$$

where $M>0$ is a constant.

Typical examples of almost sectorial operators are elliptic differential operators considered on spaces of smooth functions (e.g. Hölder continuous ones). Since this example is important in the theory of parabolic equations we recall it for completeness of presentation. 
EXAMPLE 1 . Let $\Omega \subset \mathbb{R}^{n}$ be a bounded domain with $C^{4 m}$ boundary. In the space $X=C^{\mu}(\bar{\Omega})$ of Hölder continuous functions $(\mu \in(0,1))$ consider the elliptic differential operator

$$
A v(x)=\sum_{|\beta| \leq 2 m} a_{\beta}(x) D^{\beta} v(x),
$$

with the domain $D(A)=\left\{v \in C^{2 m+\mu}(\bar{\Omega}): D^{\beta} u=0\right.$ on $\left.\partial \Omega,|\beta| \leq m-1\right\}$, where $D^{\beta}=\prod_{j=1}^{n}\left(-i \partial / \partial x_{j}\right)^{\beta_{j}}$, with $\beta$ a multiindex. The coefficients $a_{\beta}$ : $\bar{\Omega} \rightarrow \mathbb{C}$ are assumed to satisfy the following conditions:

(i) $a_{\beta} \in C^{\mu}(\bar{\Omega})$ for $|\beta| \leq 2 m$,

(ii) $a_{\beta}(x) \in \mathbb{R}$ for $|\beta|=2 m$ and all $\in \bar{\Omega}$,

(iii) $\exists_{M>0} \forall_{\xi \in \mathbb{R}^{n}} \forall_{x \in \bar{\Omega}} \quad M^{-1}|\xi|^{2 m} \leq \sum_{|\beta|=2 m} a_{\beta}(x) \xi^{\beta} \leq M|\xi|^{2 m}$.

For sufficiently large $\nu>0$ the operator $A+\nu$ is almost sectorial with parameter $\gamma=\mu / 2 m-1$. Note that in particular the minus Laplace operator satisfies the above conditions.

Observation 1 . It is easy to see that, for $v_{0} \in D(A)$ and $t>0$,

$$
\frac{d}{d t} T(t) v_{0}=\frac{1}{2 \pi i} \int_{\Gamma_{\theta}} e^{-t z}(-z)(z-A)^{-1} v_{0} d z=-T(t) A v_{0}=-A T(t) v_{0},
$$

since the operator $A$ is closed and

$$
A(z-A)^{-1}=(z-A)^{-1} A=z(z-A)^{-1}-I .
$$

As a consequence of (2.4), for each $v_{0} \in D(A)$ the function $T(t) v_{0}$ is continuous for $t \geq 0$. Indeed, integrating (2.4) over $(h, t), h>0$, we find that

$$
T(t) v_{0}-T(h) v_{0}=-\int_{h}^{t} T(s) A v_{0} d s,
$$

which shows the existence of the limit of $T(h) v_{0}$ as $h \rightarrow 0^{+}$, thanks to the estimate

$$
\begin{aligned}
\left\|\int_{h}^{t} T(s) A v_{0} d s\right\| & \leq M \int_{h}^{t} s^{-(\gamma+1)}\left\|A v_{0}\right\| d s \\
& =M\left\|A v_{0}\right\| \frac{t^{-\gamma}-h^{-\gamma}}{-\gamma} \leq M\left\|A v_{0}\right\| \frac{t^{-\gamma}}{-\gamma} .
\end{aligned}
$$

Moreover, by the resolvent identity,

$$
(z-A)^{-1}(1+A)^{-1}=(z+1)^{-1}\left((z-A)^{-1}+(1+A)^{-1}\right), \quad-1, z \in \varrho(A),
$$


hence for $v_{0} \in D(A)$ we have the equality

$$
\begin{aligned}
T(t) v_{0} & =\frac{1}{2 \pi i} \int_{\Gamma_{\theta}} e^{-t z}(z-A)^{-1} v_{0} d z \\
& =\frac{1}{2 \pi i} \int_{\Gamma_{\theta}}(1+z)^{-1} e^{-t z}(z-A)^{-1}(1+A) v_{0} d z
\end{aligned}
$$

and consequently,

$$
\begin{array}{r}
T(t) v_{0}-v_{0}=\frac{1}{2 \pi i} \int_{\Gamma_{\theta}}(1+z)^{-1}\left(e^{-t z}-1\right)(z-A)^{-1}(1+A) v_{0} d z, \\
v_{0} \in D(A),
\end{array}
$$

where the last integral is easily shown to converge to 0 as $t \rightarrow 0^{+}$. Therefore, for $v_{0} \in D(A)$, we have

$$
T(t) v_{0} \rightarrow v_{0} \quad \text { as } t \rightarrow 0^{+} .
$$

The above observation will be important when we define a classical solution to the semilinear Cauchy problem (1.1).

Consider next the linear non-homogeneous Cauchy problem

$$
w_{t}+A w=g(t), \quad w(0)=w_{0},
$$

with $g:(0, T) \rightarrow X$ and $w_{0} \in X$. When $g \in L^{1}((0, T) ; X)$ and $A$ is an almost sectorial operator, the mild solution to (2.8) is given by the Cauchy formula:

$$
w(t)=T(t) w_{0}+\int_{0}^{t} T(t-s) g(s) d s, \quad 0<t<T,
$$

where, unlike the case of $C^{0}$-semigroups, the mild solution need not be continuous at $t=0$. Recall here that the classification of semigroups according to their convergence to the identity operator as $t \rightarrow 0^{+}$has been described in the classical monograph [H-P, p. 320].

By a classical solution to (2.8) we understand a function

$$
w \in C([0, T] ; X) \cap C^{1}((0, T) ; X),
$$

which, for $0<t<T$, takes values in $D(A)$ and satisfies equation (2.8) in $X$. The following result on classical solvability of the problem (2.8) was formulated in [P-S, Theorem 4.1].

Proposition 1. If $A$ is almost sectorial and $g \in L^{\infty}((0, T) ; X)$ satisfies $g(t) \in D(A)$ for all $0<t<T$ and is Hölder continuous with exponent $\theta>\gamma+1$, that is,

$$
\|g(t)-g(s)\| \leq K|t-s|^{\theta} \quad \text { for all } 0<t, s<T,
$$

then for every $w_{0} \in \Omega$ the mild solution of (2.8) is its unique classical solution. 
Here $\Omega$ is the continuity set of the semigroup $T(t)$ :

$$
\Omega=\left\{x \in X: \lim _{t \rightarrow 0^{+}} T(t) x=x\right\} .
$$

Note that as a consequence of Observation 1 we have

$$
D(A) \subset \Omega,
$$

which gives a localization of the continuity set of the semigroup.

For completeness, we now recall some facts connected with the smoothing action of the linear semigroup $T(t), t>0$, taken from [P-S]. There, fractional powers $A^{\alpha}, \alpha \in \mathbb{C}$, of almost sectorial operators $A$ were introduced. The operators $A^{\alpha}$ are closed, injective and enjoy the characterization (limited here to real $\alpha \in(0,1)$; see [P-S, p. 52])

$$
A^{\alpha}=(1+A)^{2} \frac{1}{2 \pi i} \int_{\Gamma_{\theta}} \frac{z^{\alpha}}{(1+z)^{2}}(z-A)^{-1} d z
$$

Also the domain $D\left(A^{\alpha}\right)=X^{\alpha}$ is a Banach space endowed with the graph norm

$$
\|x\|_{X^{\alpha}}=\|x\|+\left\|A^{\alpha} x\right\|, \quad x \in X^{\alpha} .
$$

Moreover ([P-S, p. 56]), for $\beta>0$,

$$
\exists_{C=C(\gamma, \beta)} \quad\left\|A^{\beta} T(t)\right\| \leq C t^{-(\gamma+1+\beta)} .
$$

3. Semilinear problem. Consider now the solvability of the semilinear Cauchy problem (1.1) with an almost sectorial operator $A$ and $u_{0} \in \overline{D(A)}$ :

$$
u_{t}+A u=f(t, u), \quad u(0)=u_{0} .
$$

In Theorem 1 we will assume the nonlinear term $f$ to be locally Lipschitz continuous and polynomially bounded, more precisely $f:[0, \infty) \times X \rightarrow X$ is continuous with respect to $t$ and for some $1 \leq \varrho<-\gamma /(\gamma+1)$ (where $\gamma \in(-1,-1 / 2))$,

$$
\forall_{t_{0}>0} \exists_{L=L\left(t_{0}\right)} \quad\|f(t, x)-f(t, y)\| \leq L\|x-y\|\left(1+\|x\|^{\varrho-1}+\|y\|^{\varrho-1}\right),
$$

and also

$$
\forall_{t_{0}>0} \exists_{L^{\prime}=L^{\prime}\left(t_{0}\right)} \quad\|f(t, x)\| \leq L^{\prime}\left(1+\|x\|^{\varrho}\right),
$$

whenever $t \in\left[0, t_{0}\right]$.

We will be interested in mild solutions of the problem (1.1), that is, continuous solutions $u:(0, T] \rightarrow X$ of the integral equation

$$
u(t)=T(t) u_{0}+\int_{0}^{t} T(t-s) f(s, u(s)) d s .
$$

Temporarily, to avoid using the ineffective continuity set $\Omega$ of the semigroup $T(t)$ (see (2.10)), we follow [A-C-L] and search for mild solutions of 
the problem (1.1) having the same type of singularity at time $t=0$ as the linear semigroup. Therefore, for $T>0$ and fixed $\delta>0$, we introduce the metric space

$$
K\left(T, u_{0}\right)=\left\{v \in C((0, T] ; X): \sup _{t \in(0, T]}\left\|v(t)-T(t) u_{0}\right\| \leq \delta\right\}
$$

with the metric defined as

$$
\varrho_{T}\left(v_{1}, v_{2}\right)=\sup _{t \in(0, T]}\left\|v_{1}(t)-v_{2}(t)\right\| \quad \text { for } v_{1}, v_{2} \in K\left(T, u_{0}\right) .
$$

REMARK 1. As shown in Observation 1, for $u_{0} \in D(A)$ the function $[0, \infty) \ni t \mapsto T(t) u_{0} \in X$ is continuous. Therefore, for such $u_{0}$, the unique mild solution to (1.1) constructed in Proposition 2 or Theorem 1 below belongs to $C([0, \tau] ; X)$ with some $\tau \leq T$. Indeed, we can take smaller $\delta=\delta^{\prime}$ in (3.4) and obtain a unique mild solution in the metric space corresponding to $\delta^{\prime}$, possibly on a shorter time interval. But these two solutions must coincide on the common interval of existence, which proves their continuity at $t=0$.

With the above modified definition of mild solution the following simple local existence result holds. It will be useful for initial data $u_{0} \in D(A)$ in the example closing this paper.

Proposition 2. Let $A$ be an almost sectorial operator with $\gamma \in(-1,0)$ and $f$ be Lipschitz continuous on $K\left(T, u_{0}\right)$ :

$$
\begin{array}{r}
\exists_{L>0} \forall_{0<t \leq T} \forall_{x, y \in X}:\left\|x-T(t) u_{0}\right\|,\left\|y-T(t) u_{0}\right\| \leq \delta \\
\|f(t, x)-f(t, y)\| \leq L\|x-y\|,
\end{array}
$$

and bounded there:

$$
\exists_{N>0} \forall_{0<t \leq T} \forall_{x \in X:\left\|x-T(t) u_{0}\right\| \leq \delta} \quad\|f(t, x)\| \leq N .
$$

Then for sufficiently small positive $\tau_{0} \leq T$ (see (3.9)) there is a mild solution to (1.1) in $K\left(\tau_{0}, u_{0}\right)$.

Proof. We apply the Banach contraction principle in $K\left(\tau_{0}, u_{0}\right)$ to the operator

$$
F(v)(t)=T(t) u_{0}+\int_{0}^{t} T(t-s) f(s, v(s)) d s, \quad t \leq \tau_{0} .
$$

If $v \in K\left(\tau_{0}, u_{0}\right)$, then

$$
\begin{aligned}
\left\|F(v)(t)-T(t) u_{0}\right\| & \leq \int_{0}^{t}\|T(t-s)\|\|f(s, v(s))\| d s \\
& \leq M N \int_{0}^{t}(t-s)^{-(\gamma+1)} d s=M N \frac{t^{-\gamma}}{-\gamma}
\end{aligned}
$$


for $t \leq \tau_{0}$. Since the right hand side tends to 0 as $t$ goes to $0^{+}, F$ maps $K\left(\tau_{0}, u_{0}\right)$ into itself whenever

$$
M N \frac{\tau_{0}^{-\gamma}}{-\gamma} \leq \delta
$$

To finish the proof it suffices to use the estimate

$$
\begin{aligned}
\|F(v)(t)-F(w)(t)\| & \leq M \int_{0}^{t}(t-s)^{-(\gamma+1)}\|f(s, v(s))-f(s, w(s))\| d s \\
& \leq M L \frac{t^{-\gamma}}{-\gamma} \varrho_{\tau_{0}}(v, w) \quad \text { for } t \in\left[0, \tau_{0}\right],
\end{aligned}
$$

which shows that $F$ is contractive on $K\left(\tau_{0}, u_{0}\right)$ whenever $\tau_{0}>0$ is so small that

$$
M L \frac{\tau_{0}^{-\gamma}}{-\gamma}<1
$$

To satisfy both conditions imposed on $\tau_{0}$ one can set

$$
\tau_{0}=\min \left\{\left(\frac{-\gamma}{2 M L}\right)^{-1 / \gamma},\left(\frac{-\gamma \delta}{M N}\right)^{-1 / \gamma}\right\} .
$$

In further considerations the $\beta$ function will frequently be used:

$$
\beta(a, b)=\int_{0}^{1} s^{a-1}(1-s)^{b-1} d s, \quad a, b>0 .
$$

ObSERVATion 2. The boundedness condition (3.6) need not be satisfied for $u_{0} \in \overline{D(A)} \backslash D(A)$. If we replace (3.6) with the assumption

$$
\exists_{\bar{N}>0} \forall_{0<t \leq T} \quad\left\|f\left(t, T(t) u_{0}\right)\right\| \leq \bar{N}\left(1+\left\|u_{0}\right\| t^{-(\gamma+1)}\right),
$$

then the first estimate in the proof of the proposition will read:

$$
\begin{aligned}
& \left\|F(v)(t)-T(t) u_{0}\right\| \leq \int_{0}^{t}\|T(t-s)\|\|f(s, v(s))\| d s \\
& \leq M \int_{0}^{t}(t-s)^{-(\gamma+1)}\left[\left\|f(s, v(s))-f\left(s, T(s) u_{0}\right)\right\|+\left\|f\left(s, T(s) u_{0}\right)\right\|\right] d s \\
& \leq M(L \delta+\bar{N}) \frac{t^{-\gamma}}{-\gamma}+M \bar{N}\left\|u_{0}\right\| \int_{0}^{t}(t-s)^{-(\gamma+1)} s^{-(\gamma+1)} d s \\
& =M(L \delta+\bar{N}) \frac{t^{-\gamma}}{-\gamma}+M \bar{N}\left\|u_{0}\right\| t^{-2 \gamma-1} \beta(-\gamma,-\gamma) .
\end{aligned}
$$

The final expression can be made less than $\delta$ for small positive $t$ (allowing one to deduce the proposition) only when $\gamma<-1 / 2$. This observation shows 
that in further considerations the cases $\gamma \in(-1,-1 / 2)$ and $\gamma \in[-1 / 2,0)$ should be treated separately.

With assumption (3.11) replacing (3.6) the above proposition does also extend to exponents $\gamma \in[-1 / 2,0)$ provided we modify the metric space $K\left(T, u_{0}\right)$ by setting

$$
K_{\theta}\left(T, u_{0}\right)=\left\{v \in C((0, T] ; X): \sup _{t \in(0, T]} t^{\theta}\left\|v(t)-T(t) u_{0}\right\| \leq \delta\right\},
$$

with $\theta \in(2 \gamma+1,1)$. In that case the estimate corresponding to (3.12) takes the form

$$
\begin{aligned}
t^{\theta}\left\|F(v)(t)-T(t) u_{0}\right\| \leq & M L \delta t^{-\gamma} \beta(1-\theta,-\gamma) \\
& +M \bar{N} \frac{t^{\theta-\gamma}}{-\gamma}+M \bar{N}\left\|u_{0}\right\| t^{\theta-2 \gamma-1} \beta(-\gamma,-\gamma),
\end{aligned}
$$

which is sufficient to verify that $F$ maps $K_{\theta}\left(\tau_{0}, u_{0}\right)$ into itself for sufficiently small $\tau_{0}>0$. Also, the estimate corresponding to (3.8) reads

$$
t^{\theta}\|F(v)(t)-F(w)(t)\| \leq M L t^{-\gamma} \beta(1-\theta,-\gamma) \sup _{s \in\left(0, \tau_{0}\right]} s^{\theta}\|v(s)-w(s)\|
$$

whenever $t \in\left[0, \tau_{0}\right]$. The above two estimates show the existence of a fixed point of the operator $F$ in $K_{\theta}\left(\tau_{0}, u_{0}\right)$ provided $\tau_{0}>0$ is sufficiently small.

We now prove the existence of a fixed point of the operator $F$ in the metric space $K\left(\tau_{1}, u_{0}\right)$ under weaker assumptions on the nonlinear term $f$.

TheOREM 1. Let $A$ be an almost sectorial operator with $\gamma \in(-1,-1 / 2)$ and let $f$ satisfy the conditions (3.1), (3.2) with $\varrho<-\gamma /(\gamma+1)$. Then for sufficiently small positive $\tau_{1} \leq T$ there is a unique mild solution to (1.1) in $K\left(\tau_{1}, u_{0}\right)$.

Proof. We apply the Banach contraction principle in $K\left(\tau_{1}, u_{0}\right)$ to the operator

$$
F(v)(t)=T(t) u_{0}+\int_{0}^{t} T(t-s) f(s, v(s)) d s, \quad t \leq \tau_{1},
$$

with sufficiently small $\tau_{1}>0$. Note that for $v, w \in K\left(T, u_{0}\right)$ we have the estimate $\|v(t)-w(t)\| \leq 2 \delta$, and

$$
\|v(t)\| \leq \delta+M t^{-(\gamma+1)}\left\|u_{0}\right\| \quad \text { when } t \in(0, T] .
$$

Thus for $v \in K\left(\tau_{1}, u_{0}\right)$, we have

$$
\begin{aligned}
& \left\|F(v)(t)-T(t) u_{0}\right\| \leq M \int_{0}^{t}\|T(t-s)\|\|f(s, v(s))\| d s \\
& \quad \leq M L^{\prime} \int_{0}^{t}(t-s)^{-(\gamma+1)}\left[1+\left(\left\|v(s)-T(s) u_{0}\right\|+\left\|T(s) u_{0}\right\|\right)^{\varrho}\right] d s
\end{aligned}
$$




$$
\begin{aligned}
\leq & M L^{\prime} \frac{t^{-\gamma}}{-\gamma}\left(1+c \sup _{s \in(0, t]}\left\|v(s)-T(s) u_{0}\right\|^{\varrho}\right) \\
& +M^{1+\varrho} L^{\prime} c\left\|u_{0}\right\|^{\varrho} t^{-(\gamma+1) \varrho-\gamma} \beta(1-(\gamma+1) \varrho,-\gamma),
\end{aligned}
$$

with the right hand side tending to 0 as $t$ goes to $0^{+}$. Therefore $F$ maps $K\left(\tau_{1}, u_{0}\right)$ into itself whenever

$$
M L^{\prime} \frac{\tau_{1}^{-\gamma}}{-\gamma}\left(1+c \delta^{\varrho}\right)+M^{2} L^{\prime} c\left\|u_{0}\right\|^{\varrho} \tau_{1}^{-(\gamma+1) \varrho-\gamma} \beta(-(\gamma+1) \varrho,-\gamma) \leq \delta,
$$

where, to ensure the above inequality for small $\tau_{1}>0$, we have used the condition $\varrho<-\gamma /(\gamma+1)$.

To finish the proof it suffices to show that $F$ is contractive on $K\left(\tau_{1}, u_{0}\right)$ when $\tau_{1}>0$ is sufficiently small. Indeed, for $v, w \in K\left(\tau_{1}, u_{0}\right)$,

$$
\begin{aligned}
& \|F(v)(t)-F(w)(t)\| \\
\leq & M L \int_{0}^{t}(t-s)^{-(\gamma+1)}\|v(s)-w(s)\|\left(1+\|v(s)\|^{\varrho-1}+\|w(s)\|^{\varrho-1}\right) d s \\
\leq & \varrho t(v, w)\left(M L \frac{t^{-\gamma}}{-\gamma}+2 M L \int_{0}^{t}(t-s)^{-(\gamma+1)}\left(\delta+M t^{-(\gamma+1)}\left\|u_{0}\right\|\right)^{\varrho-1} d s\right) \\
\leq & \varrho_{\tau_{1}}(v, w) M L\left(\left(1+2 c \delta^{\varrho-1}\right) \frac{\tau_{1}^{-\gamma}}{-\gamma}\right. \\
& \left.+2 c\left(M\left\|u_{0}\right\|\right)^{\varrho-1} \tau_{1}^{-(\gamma+1) \varrho+1} \beta(1-(\gamma+1)(\varrho-1),-\gamma)\right)
\end{aligned}
$$

whenever $0<t \leq \tau_{1}$. Since the expression in the last bracket tends to 0 as $\tau_{1}$ goes to $0^{+}$, the claim follows. The solution obtained is unique.

REMARK 2. As usual the local solution constructed above may be extended to a solution defined on a maximal interval of existence. We will not formulate the corresponding result rigorously.

3.1. More regular solutions. We will use the theory of the linear nonhomogeneous equation (2.8) reported in Proposition 1 to find conditions allowing the mild solutions of (1.1) constructed in Theorem 1 to be more regular; in fact, to be classical solutions. The latter means that

$$
u \in C([0, T] ; X) \cap C^{1}((0, T) ; X),
$$

$u$ has values in $D(A)$ for all $t \in(0, T)$ and satisfies (1.1) in $X$. Before formulating the announced theorem we need some preparation.

REMARK 3. As shown in (2.4), for $v_{0} \in D(A)$ and $t>0$,

$$
T(t) A v_{0}=A T(t) v_{0} .
$$


It follows that for fixed $v_{0} \in D(A)$ the left hand side above is continuous from $(0, T]$ into $X$ with singularity at $t=0^{+}$of order $t^{-(\gamma+1)}$. Thus, for such $v_{0}, T(t) v_{0}$ is continuous from $(0, T]$ into $D(A)$ with the same type of singularity at $t=0^{+}$.

If we assume that $v_{0} \in D(A)$ and $A v_{0} \in \Omega$ (the continuity set of the semigroup $T(t)$, see (2.10)), then

$$
T(t) A v_{0} \rightarrow A v_{0} \quad \text { as } t \rightarrow 0^{+} .
$$

These two properties together show that for $v_{0}$ belonging to the set

$$
\{v \in D(A): A v \in \Omega\},
$$

the function $[0, T] \ni t \mapsto T(t) v_{0} \in D(A)$ is continuous. Note also that, due to the localization of $\Omega$ given in (2.11), the set (3.16) contains $D\left(A^{2}\right)$.

Our next task will be to discuss solvability of the problem (1.1) in the space $D(A)$ of smooth functions. For $u_{0} \in D(A)$ and $\delta^{\prime}>0$ set

$$
\widetilde{K}\left(\tau_{2}, u_{0}\right)=\left\{v \in C\left(\left(0, \tau_{2}\right] ; D(A)\right): \sup _{t \in\left(0, \tau_{2}\right]}\left\|v(t)-T(t) u_{0}\right\|_{D(A)} \leq \delta^{\prime}\right\} .
$$

Proposition 3. Let $A$ be an almost sectorial operator with $\gamma \in$ $(-1,-1 / 2), u_{0} \in D(A)$ and let $f$ satisfy the condition

$$
\begin{aligned}
\exists_{\widetilde{L}>0} \forall_{0<t \leq T} \forall_{x, y \in D(A)} & \left(\left\|x-T(t) u_{0}\right\|_{D(A)},\left\|y-T(t) u_{0}\right\|_{D(A)} \leq \delta^{\prime}\right) \\
\Rightarrow & \|f(t, x)-f(t, y)\|_{D(A)} \leq \widetilde{L}\|x-y\|_{D(A)},
\end{aligned}
$$

and also

$$
\exists_{\widetilde{N}>0} \forall_{0<t \leq T} \quad\left\|f\left(t, T(t) u_{0}\right)\right\|_{D(A)} \leq \tilde{N}\left(1+\left\|u_{0}\right\|_{D(A)} t^{-(\gamma+1)}\right) .
$$

Then, for sufficiently small positive $\tau_{2} \leq T$, there is a unique mild solution to (1.1) in $\widetilde{K}\left(\tau_{2}, u_{0}\right)$.

The proof of this proposition, analogous to the proof of the extension of Proposition 2 reported in Observation 2, will be omitted. Regular solutions constructed in this proposition will be called $D(A)$-smooth solutions.

We are now able to formulate the main result of this section. See Theorem 1.6 of [PA, Chapter 6] for a similar result concerning $C^{0}$-semigroups.

Theorem 2. Suppose that $-1<\gamma<-1 / 2, u_{0} \in D(A)$ with $A u_{0} \in \Omega$ and $u$ is the mild solution corresponding to $u_{0}$, existing on $\left[0, \tau_{1}\right]$. Suppose the nonlinearity $f$ satisfies the condition

$$
\begin{aligned}
\exists_{L=L(N)} \forall_{x, y \in X,\|x\|,\|y\| \leq N} \forall_{t, s \in[0, T]} \\
\|f(t, x)-f(s, y)\| \leq L\left(|t-s|^{\theta}+\|x-y\|\right)
\end{aligned}
$$

with an exponent $\theta>\gamma+1$, and the assumptions of Proposition 3. Then $u$ is in fact the unique classical solution of (1.1), possibly on a shorter time interval. 
Proof. According to Proposition 1, it suffices to show that $f(t, u(t)) \in$ $D(A)$ for $t \in\left(0, \tau_{3}\right)$ (with some $\left.\tau_{3} \leq \tau_{1}\right)$, and that the mild solution $u$ is in $C^{\beta}\left(\left(0, \tau_{1}\right) ; X\right)$ with some $\beta>\gamma+1$.

Note first that under our extra assumptions on the initial data $u_{0}$, by Proposition 3, there is a unique $D(A)$-smooth solution corresponding to $u_{0}$. Evidently, by uniqueness, the mild and $D(A)$-smooth solutions coincide on the common interval of existence $\left[0, \min \left\{\tau_{1}, \tau_{2}\right\}\right]$. This proves the first property with $\tau_{3}=\min \left\{\tau_{1}, \tau_{2}\right\}$.

Now we show that thanks to assumption (3.18) the mild solution $u$ belongs to $C^{\beta}\left(\left(0, \tau_{1}\right) ; X\right)$ with some $\beta>\gamma+1$. Note that when $u_{0} \in D(A)$, the function $T(t) u_{0}$ is continuous for $t \geq 0$ and the corresponding mild solution $u$ is bounded in $X$ for $t \in\left[0, \tau_{1}\right]$. For small positive $h_{0}$ and $t \in\left(0, \tau_{1}-h_{0}\right]$, $0<h<h_{0}$, we will estimate the difference

$$
\begin{aligned}
& =T(t+h) u_{0}-T(t) u_{0}+\int_{0}^{t+h} T(t+h-s) f(s, u(s)) d s-\int_{0}^{t} T(t-s) f(s, u(s)) d s \\
& =T(t)\left[T(h) u_{0}-u_{0}\right]+\int_{0}^{t} T(t-s)[f(s+h, u(s+h))-f(s, u(s))] d s \\
& \quad+\int_{0}^{h} T(t+h-s) f(s, u(s)) d s .
\end{aligned}
$$

Thanks to (3.18), for $N$ so large that $\|u(t)\| \leq N$ for $t \in\left[0, \tau_{1}\right]$,

$$
\begin{aligned}
& \int_{0}^{t}\|T(t-s)\|\|f(s+h, u(s+h))-f(s, u(s))\| d s \\
& \leq M L(N) \int_{0}^{t}(t-s)^{-(\gamma+1)}\left(|h|^{\theta}+\|u(s+h)-u(s)\|\right) d s \\
& \leq M L(N) \frac{t^{-\gamma}}{-\gamma}|h|^{\theta}+M L(N) \int_{0}^{t}(t-s)^{-(\gamma+1)}\|u(s+h)-u(s)\| d s,
\end{aligned}
$$

and, for $m^{\prime}$ such that $\|f(t, u(t))\| \leq m^{\prime}$ for $t \in\left[0, \tau_{1}\right]$,

$$
\int_{0}^{h}\|T(t+h-s)\|\|f(s, u(s))\| d s \leq M m^{\prime} \frac{(t+h)^{-\gamma}-t^{-\gamma}}{-\gamma} .
$$

Finally, as follows from Observation 1,

$$
\left\|T(t)\left[T(h) u_{0}-u_{0}\right]\right\| \leq M\left\|A u_{0}\right\| \int_{t}^{t+h} s^{-(\gamma+1)} d s=M\left\|A u_{0}\right\| \frac{(t+h)^{-\gamma}-t^{-\gamma}}{-\gamma} .
$$


Collecting the estimates, using the inequality $(t+h)^{-\gamma}-t^{-\gamma} \leq h^{-\gamma}$, $-\gamma \in(1 / 2,1)$, we find that

$$
\begin{aligned}
& \|u(t+h)-u(t)\| \leq\left(M\left\|A u_{0}\right\|+M m^{\prime}\right) \frac{|h|^{-\gamma}}{-\gamma} \\
& +M L(N) \frac{\tau_{1}^{-\gamma}}{-\gamma}|h|^{\theta}+M L(N) \int_{0}^{t}(t-s)^{-(\gamma+1)}\|u(s+h)-u(s)\| d s .
\end{aligned}
$$

Since $-\gamma>\gamma+1$ for $-\gamma \in(1 / 2,1)$, setting $\beta=\min \{\theta,-\gamma\}>\gamma+1>0$, we arrive at

$$
\frac{\|u(t+h)-u(t)\|}{|h|^{\beta}} \leq \mathrm{const}+M L(N) \int_{0}^{t}(t-s)^{-(\gamma+1)} \frac{\|u(s+h)-u(s)\|}{|h|^{\beta}} d s .
$$

The usual Gronwall lemma then applies and we find that

$$
\frac{\|u(t+h)-u(t)\|}{|h|^{\beta}} \leq \text { const } \cdot \exp \left(M L(N) \frac{t^{-\gamma}}{-\gamma}\right) \leq \text { const }^{\prime}
$$

for $0<t \leq \tau_{1}-h_{0}$ and $0<h<h_{0}$. The proof is thus completed.

3.2. An example. Finally, we give a simple example illustrating the presented theory. Since there is no significant difference in considerations for the general elliptic operator described in Example 1 and the basic Laplace operator with Dirichlet boundary condition, we will consider a simple problem

$$
\left\{\begin{array}{l}
u_{t}=\Delta u+f(u), \quad t>0, \quad x \in \Omega, \\
u(0, x)=u_{0}(x) \quad \text { in } \Omega, \quad u=0 \text { on } \partial \Omega .
\end{array}\right.
$$

Let $\mu \in(0,1)$ be the Hölder exponent and $\Omega \subset \mathbb{R}^{n}$ be a bounded domain with $\partial \Omega \in C^{4}$. In the space $C^{\mu}(\bar{\Omega})$ of Hölder continuous functions consider the extension $A$ of $-\Delta$ with the domain

$$
D(-\Delta)=\left\{v \in C^{2+\mu}(\bar{\Omega}): v=0 \text { on } \partial \Omega\right\} .
$$

The operator $A: D(A) \subset C^{\mu}(\bar{\Omega}) \rightarrow C^{\mu}(\bar{\Omega})$ is almost sectorial with exponent $\gamma=\mu / 2-1 \in(-1,-1 / 2)$, but not sectorial (see [P-S, p. 44]). Note also that $A$ is not densely defined since

$$
D(A) \subset\left\{v \in C^{\mu}(\bar{\Omega}): v=0 \text { on } \partial \Omega\right\},
$$

which is not dense in $C^{\mu}(\bar{\Omega})$.

Recall that $C^{\mu}(\bar{\Omega}), \mu \in(0,1)$, is a Banach space of uniformly continuous functions $v: \bar{\Omega} \rightarrow \mathbb{R}$ equipped with the norm

$$
\|v\|_{\mu}=\sup _{x \in \bar{\Omega}}|v(x)|+\sup _{x, y \in \bar{\Omega}, 0<|x-y| \leq 1} \frac{|v(x)-v(y)|}{|x-y|^{\mu}}=\|v\|_{C(\bar{\Omega})}+H^{\mu}(v) .
$$


A sufficient condition for a real function $f: \mathbb{R} \rightarrow \mathbb{R}$ to generate a bounded and locally Lipschitz substitution (Nemytskiॅ) operator $\mathcal{F}: C^{\mu}(\bar{\Omega}) \rightarrow C^{\mu}(\bar{\Omega})$,

$$
\left\|\mathcal{F}\left(x_{1}\right)-\mathcal{F}\left(x_{2}\right)\right\|_{\mu} \leq k(r)\left\|x_{1}-x_{2}\right\|_{\mu}, \quad\left\|x_{1}\right\|_{\mu},\left\|x_{2}\right\|_{\mu} \leq r,
$$

was formulated in [A-Z, Theorem 7.9] (see also the remark on p. 204 there). To ensure (3.23) we need to assume that $f$ is continuously differentiable on $\mathbb{R}$ with

$$
\left|f^{\prime}(x)-f^{\prime}(y)\right| \leq k(r) \frac{|x-y|}{r}, \quad|x|,|y| \leq r .
$$

If, in Proposition 2, we take smooth initial data $u_{0} \in D(A)$ then, according to Remark 1 , the function $T(t) u_{0}$ is continuous at $t=0^{+}$and the values of functions in $K\left(T, u_{0}\right)$ vary in a bounded subset of $C^{\mu}(\bar{\Omega})$. Assuming (3.24), thanks to (3.23), Proposition 2 then provides a unique mild solution for all $u_{0} \in D(A)$. Existence of mild solutions to (3.22) for such $u_{0}$ is thus justified.

If we assume additionally that $f^{\prime}, f^{\prime \prime}: \mathbb{R} \rightarrow \mathbb{R}$ are continuously differentiable functions satisfying condition (3.24), then a simple but lengthy calculation using the fact that $C^{\mu}(\bar{\Omega})$ is a Banach algebra shows that the assumptions of Proposition 3 are satisfied. Thus, for $u_{0} \in D(A)$ with $A u_{0} \in \Omega$ and $f$ as described, the corresponding mild solution to (3.22) is also a classical solution.

\section{References}

[A-Z] J. Appell and P. P. Zabrejko, Nonlinear Superposition Operators, Cambridge Univ. Press, Cambridge, 1990.

[A1] W. Arendt, Contributions to the theory of semigroup distributions, Ph.D. Dissertation, Univ. Antwerpen, 1983.

[A2] - Vector valued Laplace transforms and Cauchy problems, Israel J. Math. 59 (1987), 327-352.

[A-C-L] J. M. Arrieta, A. N. Carvalho and G. Lozada-Cruz, Dynamics in dumbbell domains II. The limiting problem, in preparation.

[FR] A. Friedman, Partial Differential Equations of Parabolic Type, Prentice-Hall, Englewood Cliffs, NJ, 1964.

[H-P] E. Hille and R. S. Phillips, Functional Analysis and Semi-groups, Amer. Math. Soc., Providence RI, 1957.

[H] L. Hörmander, Estimates for translation invariant operators in $L^{p}$ spaces, Acta Math. 104 (1960), 93-139.

[K-H] H. Kellerman and M. Hieber, Integrated semigroups, J. Funct. Anal. 84 (1989), $160-180$.

[N-S] J. M. A. M. van Neerven and B. Straub, On the existence and growth of mild solutions of the abstract Cauchy problem for operators with polynomially bounded resolvent, Houston J. Math. 24 (1998), 137-171.

[PA] A. Pazy, Semigroups of Linear Operators and Applications to Partial Differential Equations, Springer, New York, 1983. 
[P] F. Periago, Global existence, uniqueness, and continuous dependence for a semilinear initial value problem, J. Math. Anal. Appl. 280 (2003), 413-423.

[P-S] F. Periago and B. Straub, A functional calculus for almost sectorial operators and applications to abstract evolution equations, J. Evol. Equations 2 (2002), 41-68.

[T] H. R. Thieme, "Integrated semigroups" and integrated solutions to abstract Cauchy problems, J. Math. Anal. Appl. 152 (1990), 416-447.

Tomasz Dlotko

Institute of Mathematics

Silesian University

40-007 Katowice, Poland

E-mail: tdlotko@ux2.math.us.edu.pl

Received March 7, 2007;

received in final form September 4, 2007 